\title{
L'insurrezione erzegovese. La Slavia - Il mondo di Bonaventura Vidovich tra letteratura, mitologia e storia
}

\author{
Ana Bukvić \\ Università di Zara, Dipartimento di Italianistica
}

Prispevek prinaša analizo drame Linsurrezione erzegovese. La Slavia - Il mondo Bonaventure Vidovicha, ki je izšla v Livornu leta i875, ko je vladalo posebno zanimanje za bosansko vprašanje; v ta sklop zanimanja se uvršča tudi tragedija Luigija Ficherta Bielka di Bosnia (Bjelka iz Bosne). Prispevek analizira Vidovichevo prepletanje med literaturo, mitologijo in zgodovino, s katerim želi ustvariti kulturno okolje, ki bi izbrisalo vsako delitev med narodi in ljudstvi kot so Italijani, Slovani in Turki v zapletenem zgodovinskem kontekstu.

Ključne besede: Bonaventura Vidovich, L'insurrezione erzegovese. La Slavia - Il mondo, literarno okolje, alegorično-socialna drama

The contribution analyses the social-allegoric drama Linsurrezione erzegovese. La Slavia - Il mondo of Bonaventura Vidovich, published in Livorno in I875. It is a period of the particular interest for the Bosnian social and political context, which can be seen also in Fichert's tragedy Bielka di Bosnia. The contribution tries to highlight how Vidovich uses literature, mythology and history to build a cultural imaginary that erases all limits between nations and peoples such as the Italians, the Slavs and the Turks in a complex historical context.

Key words: Bonaventura Vidovich, Linsurrezione erzegovese. La Slavia - Il mondo, cultural imaginary, social-allegorical drama

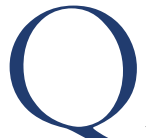

uesto contributo verte sull'analisi dell'intersezione tra letteratura, mitologia e storia nel dramma L'insurrezione erzegore. La Slavia - Il mondo di Bonaventura Vidovich, figlio unico di Anna e Marco Antonio Vidovich, noti letterati e intellettuali dalmati ${ }^{\mathrm{I}}$. Sappiamo poco della sua vita. È, infatti, la corrispondenza tra i coniugi Vidovich e Niccolò Tommaseo che mette in luce il profilo del giovane Bonaventura ${ }^{2}$. Si suppone che Bo-

I Per una biografia dettagliata rinvio agli studi di: Zorić 1970, 1971; Dujmić 1998; Rados 2007; Tomasović 2009; Nižić 2009.

2 I coniugi Vidovich conobbero Niccolò Tommaseo il 25 ottobre I8 39 sul piroscafo del Lloyd austriaco "Barone Stürmer" durante il viaggio tra Curzola, Ragusa e Cattaro nell'anno in cui Tommaseo riscopriva la Dalmazia. Oltre ad essere amici, i coniugi Vidovich naventura sia nato intorno al 1828 (Zorić 2002, 367-54I) e Marco Antonio Vidovich diede a suo figlio il nome di suo fratello Bonaventura Vidovich (1775-1859). ${ }^{3}$ In una delle lettere indirizzate a Tommaseo, Marco Antonio Vidovich cer-

e Niccolò Tommaseo collaboravano a un progetto di raccolta dei canti popolari dalmati (Zorić 2002,367-54I).

3 Durante il dominio francese, Bonaventura Vidovich, fratello di Marco, era segretario del cavaliere Giandomenico Garagnino a Ragusa (Dubrovnik), e in seguito rimase nel servizio civile, occupandosi principalmente di questioni economiche. Nel I84I, il pretore di Sebenico, Natali, informò la polizia di Zara che si trattava di un buon funzionario, affidabile e diligente, fedele alle autorità austriache. Tommaseo lo descrisse nel modo seguente: "Uomo nobile d'animo, ancor, più che d'origine, cortese, ingegnoso, operoso, che faticò tutta la vita soffrendo disagi in pro della sua numerosissima e tutta ingegnosa famiglia" (Zorić 2002, 421). Nel I 847 Giuseppe Sabalich lo chiama dilettante filarmonico accanto a Simeone 
ca una raccomandazione per il figlio e accenna al suo carattere pesante:

$$
\begin{aligned}
& \text { Com'Ella sà ho un figlio. Lode al Cielo non è } \\
& \text { stupido, ma si bene piuttosto intollerante del } \\
& \text { fermo oppressivo sistema dell'odierna pub- } \\
& \text { blica istruzione. Quindi ha dovuto quest'an- } \\
& \text { no ripetere la prima Ginnasiale. Ben spe- } \\
& \text { ro che quest'anno lo supererà questo colle } \\
& \text { di bronchi e sterpi. In ogni modo gli torne- } \\
& \text { rebbe sempre ad utile una di Lei raccoman- } \\
& \text { dazione al Sig. Professor Butturi, Direttore } \\
& \text { dei Ginnasi.- Per tale raccomandazione ap- } \\
& \text { punto a Lei ricorro.- Se la ricerca sta in con- } \\
& \text { venienza ai di Lei riguardi, mi porgierò di } \\
& \text { consegnare io stesso la lettera, che Ella può } \\
& \text { spedirmi liberalmente con la posta.- Mi per- } \\
& \text { doni, e mi seguiti la Sua grazia (Zorić } 2002 \text {, } \\
& \text { 526-27). }
\end{aligned}
$$

È interessante il rapporto della polizia di Zara del 7 luglio I844, in cui si afferma che Marco Vidovich abbia un figlio di circa 16 anni che adora i bar e il biliardo e frequenti Giulio Sabalic (ibi, 453). Dopo la scomparsa del marito, avvenuta nel 1868, Anna Vidovich ha informato Tommaseo che suo figlio si era trasferito in Italia. Bonaventura Vidovich sperava di concretare facilmente le sue idee letterarie e filantropiche in una nuova atmosfera di democrazia e con l'aiuto di Niccolò Tommaseo, Federico Seismit-Doda ed Emilio de Tipaldo. La preoccupazione e il dolore per la perdita del figlio e dei nipoti è stata espressa da Anna Vidovich anche in versi che sono conservati in un manoscritto nel Fondo Tommaseo a Firenze 4 . Dopo l'insuccesso in Italia, Bonaventura è riuscito a trovare una posizione di segretario comunale a Scardona in Dalmazia con l'aiuto del famoso pittore zaratino Francesco Salghetti Drioli (Zorić 2002, 52I). La produzione letteraria di Bonaventura Vidovich è di breve respiro, poco capace di creare una linea di sviluppo, cioè di dare continuità allo slan-

de Stermich, Amadeo Fabris, Carlo Zerboni e altri (Sabalich 1904I922, I8I).

4 "Piangi il consorte! / pe' figli cari / Pietà richiedi misera Madre! / Ah! ti conforta, va sì chi or vole / Esser de' figli tuoi anche il padre!" (Zorić 2002,521). cio creativo. In allegato alla lettera del 25 marzo 1844, Marco Antonio Vidovich inviò a Tommaseo alcuni componimenti del figlio in prosa $e$ in versi, giustificando i suoi errori grammaticali e stilistici con un accenno a lezioni deludenti in verseggiatura. Nomina una sessantina dei lavori del giovane Bonaventura definendoli dram$\mathrm{mi}$, commedie e farse che purtroppo sono rimasti indisponibili (Zorić 2002, 526-527). Poche opere sono state pubblicate: Idee accennanti al modo di togliere il pauperismo presentate ai filantropi ricchi, agiati e poveri, un saggio pubblicato a Livorno nel $1876^{5}$, la poesia In morte di S.M. il re d'Italia Vittorio Emanuele II e per l'avvenimento al trono di S.M. il re Umberto I nel 1878 (Vidovich 1878) e il dramma allegorico-sociale L'insurrezione erzegovese. La Slavia - Il mondo in sei atti con prologo, pubblicato sempre a Livorno nel 1875 e dedicato agli amici dell'umanità. La notizia del dramma allegorico-sociale era apparsa sulla rivista zagabrese Vienac il is dicembre 1875 e fu Ivan August Kaznačić a comunicare che il figlio di Anna Vidovich ${ }^{6}$ era autore di un dramma allegorico-sociale, Ercegovački ustanak. Slavija. Sviet., il cui valore letterario non si poteva svalutare per il fine umanitario dell'opera (Kaznačić i876, 79-80). Il dramma incuriosì Giuseppe Garibaldi, fatto che veniamo a sapere da una lettera del famoso condottiero e patriota italiano indirizzata a Bonaventura nel 1875 (Berlam 1934). L'interesse per la Bosnia e Erzegovina di Bonaventura Vidovich trova la sua origine nella tradizione familiare. Delle sciagure del popolo bosniaco aveva scritto già suo padre Marco Antonio Vidovich nel I844. Egli tradusse la poesia Ercegovci i Bošnjaci. Çastnomu gospodinu A. Gutti na njegovu xalbu koju pokazuje u Zori pod brojem 30 tekuche godine pubblicata nel-

$5-\quad$ In questa discussione, in dieci capitoli, mostra una sincera preoccupazione per i gravi problemi socio-economici che affliggevano la società e gli individui allo stesso tempo. Nella prefazione, sottolinea che è abituato a pensare di voler scrivere come pensa, mentre il suo obiettivo è il bene comune, e aggiunge che si sforza di andare oltre i confini della convivenza e dei diritti. Dall'ampio testo in prosa apprendiamo che è contro la violenza, il politicismo e le false promesse. Ai poveri dovrebbe essere data l'opportunità di ricevere l'istruzione ed avere un lavoro (Zorić 2002, 526-527). Ivan August Kaznačić per errore dice "il fu Ana Vidovich”, però la famosa scrittrice dalmata morì quattro anni dopo, nel 1879 . 
la Zora dalmatinska nel i848. Il titolo della traduzione era I popoli d'Ercegovina e Bosna ad Antonio Gutta pel suo lamento nella Zora al n. 20 anno corrente. Versione dall'illirico nella Zora al n. 4o. Dedicata alle Guardie Nazionali Dalmate, Zara, 1848. L'introduzione alla traduzione venne fatta da Luigi Giurich. Marco Antonio Vidovich scrisse della necessità che gli slavi dalmati aiutassero il popolo fraterno che soffriva sotto il giogo straniero (Zorić 1961, I88), mentre uno dei motivi per la stesura dell'opera può essere l'origine bosniaca della famiglia Vidovich (Vidovich I875, I).

\section{I}

Il titolo del dramma L'insurrezione erzegovese. La Slavia - Il mondo ci dirige inesorabilmente al dramma storico come punto di partenza. La rivolta, iniziata dai Croati, si svolse nel 1875 in Erzegovina, soffocata, però, dall'Impero ottomano, che poi fu sconfitto dalla Russia e dovette accordarsi per la pace a Santo Stefano, e più avanti per un nuovo trattato di pace a Berlino nel I878, con il quale la Bosnia e Erzegovina furono incluse nel protettorato austro-ungarico ${ }^{7}(\mathrm{~Pa}-$ vličević 2000, I2). L'anno di pubblicazione del dramma coincide con l'anno della rivolta e ne illumina la prospettiva storica, ma Vidovich non ha presente l'esito della rivolta, così lo sfondo storico non è esplicitamente esposto. Egli, invece, privatizza un conflitto sociale e lo rappresenta attraverso un intreccio amoroso, il che serve da incitamento alla rivolta. Il dramma consta di 6 atti e presenta un'ampia panoramica dei personaggi che contribuisce a conferire al dramma una forma aperta (Pfister 1998, 246). Ci sono Mohamed-Pascià, governatore d'Erzegovina, Fatima, una giovane turca, moglie del Pascià, Ahmed, colonnello e comandante delle truppe turche, Djemal, un giovane capitano turco, l'addetto al Pascià, Paolo, giovane possidente erzego-

\footnotetext{
Le fonti storiche testimoniano che il periodo degli anni Sessanta fu un periodo di pace e di lavoro per merito del visir Topal Šerif Osman-Paša, l'ultimo gran visir della Bosnia che assunse questo incarico dal ı 860 al 1869 . Dagli anni Settanta, con l'arrivo di Safvet Paša nel i869, cominciano degli anni tormentosi contrassegnati da insurrezioni e da un' intolleranza cristiano-musulmana (Pavličević 2000, I2)
}

vese, Caterina, signora erzegovese, sua figlia $\mathrm{Da}$ niza, Giorgio, signore montenegrino, Adalberto, giovane signore italiano, fra Guido, cappuccino di circa 60 anni, un oste, il servo di Caterina, il Negro, due schiave turche, schiavi neri, il popolo erzegovese, soldati turchi, i personaggi del prologo, il personaggio allegorico rappresentante la fede. L'azione è ambientata a Trebinje, in Erzegovina. In un instante ci troviamo di fronte a uno spazio dilatato, in cui viene a situarsi un ampio numero di personaggi, ma Vidovich sembra essere attratto più dalla dimensione dell'interno che di quella dell'esterno. Ė solo nel prologo e nella scena finale che presenta un ambiente aperto. Nel prologo l'autore menziona una campagna e un fiume che potrebbe essere Trebišnjica, il fiume che scorre per Trebinje. Si nota un'equivalenza semantica tra il luogo dell'azione e le vicende. Gli spazi chiusi sono presentati come spazi che generano ansia ed angoscia: "Fatim. [...] Già, così rinchiusa come sono, in un dorato e gemmato sepolcro, la mia esistenza è inutile [...]" (Vidovich 1875,72$)$, mentre gli spazi non limitati, cioè aperti, hanno una valorizzazione positiva e creano l'ambiente per le nozze di Paolo e Daniza e la scena del prologo dove incontriamo il Destino delle Nazioni e la Vila.

Il prologo derealizza che il titolo implicasse una vicenda storica precisa e sottintendesse una ricostruzione dell'episodio storico. Inoltre, le dramatis personae del prologo incarnano le virtù e le passioni e contribuiscono all'allegoria del dramma (Solar 20II, I3). Sono Il Destino delle Nazioni; un uomo d'imponente aspetto con una spada, La Vila rappresentata da una giovinetta in costume slavo con berretto rosso sul capo e $\mathrm{i}$ capelli sciolti; la guzla in mano, e i cinque geni rappresentanti delle diverse razze, ossia: la razza bianca, la razza tartaro-asiatica, la razza americana, la razza malese e la razza nera. Il prologo è ambientato in campagna tra le colline dove scorre il fiume sulle cui sponde è seduta la Vila, figura della mitologia slava. È notte e si sente il suono delle melodie tristi che la Vila vuole accompa-

8 Si tratta dello strumento musicale slavo simile alla viola, provvisto di una sola corda che si fa vibrare mediante un arco. 
gnare con le guzle. Si rivolge al Creatore dal quale cerca la pace e l'amore. La consola Il Destino delle Nazioni che si avvicina a lei:

[...] Ma tu, o Vila, degli Slavi, tu piangevi po-
canzi, come piangi da lunghi anni, ogni sera,
sulle sorti degli Slavi, tuoi spirituali fratel-
li. Piangesti assai, ed assai, di mesti canti agli
Slavi amanti e guerrieri inspirasti! Or basta!
Fra gli essere dello spazio che influiscono e
vegliano; per voler del Supremo Spirito, su-
gli uomini, guidandoli per aspre vie alla meta
del bene e dell'ordine, primo son' io, ed io ti
dico, o Vila, per gli Slavi il giorno di pace ver-
rà (Vidovich 1875,6 ).

Inoltre, invita con la spada i cinque geni uniti dal nodo spirituale della fratellanza, sperando così l'unione di altre nazioni della terra. $\mathrm{Li}$ invia verso i loro pianeti a diffondere la fratellanza per mezzo dell'umanità e della scienza. L'insurrezione erzegovese condivide con i drammi allegorici il comune desiderio di dare una lezione didattica sul giusto e sull'ingiusto. Zorić afferma che lo scrittore era un appassionato sostenitore delle comprensioni spiritistiche, unite ad elementi delle scienze naturali. Gli Slavi e le altre nazioni raggiungeranno uno stato perfetto di pace ed allegria con l'aiuto della collaborazione spirituale con il Destino delle Nazioni, però dopo molto dolore e sofferenza, cosa che si nota nei personaggi del dramma che discutono sul diritto di una vita libera e di fratellanza con altri popoli (Zorić 1961, I89). Da questa cornice esce il personaggio di Paolo, fidanzato di Daniza, che chiede vendetta per Daniza e Caterina e morte ai tiranni e ai despoti, però fra Guido gli risponde nel modo seguente:

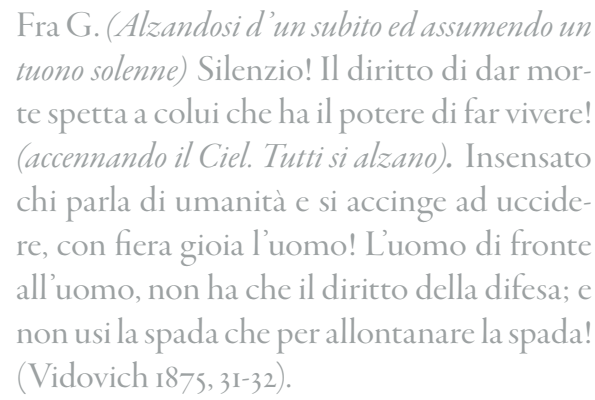

Nella scena d'apertura appaiono Daniza e fra Guido in confessione nel convento; in questa scena, pur cercando di negare l'amore verso Djemal, giovane capitano turco e musulmano, Daniza, si rivela innamorata di lui. D'un lato lo scenario storico-politico ostacola il loro amore:

\section{D: Dunque, padre mio, noi dobbiamo per- donare ai Turchi tutte le loro atrocità passa- te e presenti? Le estorsioni, gli incendi, i rapi- menti, le morti? ... Tutto tutto! ... (Vidovich I875, I0)}

e dall'altro Paolo, il fidanzato di Daniza, insieme a Giorgio, signore montenegrino, viene nel convento a pianificare la guerra "per la santa causa" (ibi, I4). Alla replica finale di Giorgio:

$$
\begin{aligned}
& \text { I veri Slavi, e gli uomini tutti che hanno cuo- } \\
& \text { re, intelligenza, ed amore pel proprio simile, } \\
& \text { non lo faranno, e la vittoria potrà essere ritar- } \\
& \text { data, ma non impedita! Coraggio! Vi è una } \\
& \text { forza più possente di ogni altra: il diritto di } \\
& \text { natura, sentito dai popoli oppressi! (ibid.) }
\end{aligned}
$$

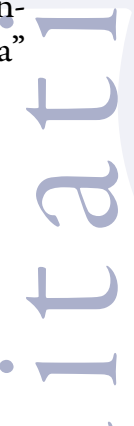

il frate risponde: “[...] Vi è una forza più possente ancora: la giustizia di Dio!” (ibid.)

Nel secondo atto l'azione si sposta nella casa di Caterina. Djemal, travestito da erzegovese, va a trovare Daniza e le dice:

$$
\begin{aligned}
& {[\ldots . .] \text { ma so ancora che amore non conosce ri- }} \\
& \text { guardi, non ha ritegni, perché amore è un } \\
& \text { sentimento ardente e nobile, che, non cer- } \\
& \text { cato da noi mortali, ci viene ispirato da Al- } \\
& \text { lah! Non è tua colpa né tuo merito, o Dani- } \\
& \text { za, l'essere Cristiana, come non è mia colpa } \\
& \text { né merito l'essere Maomettano (ibi, I5). }
\end{aligned}
$$

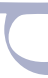

Infatti, tramite Djemal, Bonaventura Vidovich enfatizza l'immagine positiva dei Turchi, fatto che attesta la distanza tra le rappresentazioni imagotipiche e la realtà empirica, cosa che si evince dalla seguente citazione:

\footnotetext{
Djemal. [...] Il Turco è feroce, lo so, lo sa il mondo; ma anche fra i Turchi vi sono cuori che sentono, che comprendono, che amano; ed anche il popolo di Maometto, come ogni altro popolo, sarebbe suscettibile di coltura
} 
eperfezionamento, se fra i grandi meglio regnassero giustizia ed ordine! [...] Non accusare, o Daniza, coloro che obbediscono al più imperiose e assoluto, e minaccioso comando. Accusa pure, ed odia coloro che impongono! Odia, Daniza, odia con me! ... (ibi, 17)

Nel finale del secondo atto, Daniza lo definisce uomo di cuore e conferma il loro affetto reciproco che contribuisce alla suspense drammatica.

Nel terzo atto si presenta Mohamed Pascià, convinto che il popolo non si sarebbe organizzato in una rivolta. Egli esprime tristezza a sentire della propria crudeltà, il che provoca una reazione in un discorso a parte nel quale Ahmed lo vede come furbo, Djemal lo chiama "ipocrita”, Paolo "il vile tiranno", mentre Giorgio chiude un discorso a parte con "schifoso rettile". Proprio in questa scena si accentua il discorso a parte che Vidovich usa nella caratterizzazione dei personaggi. Una spiegazione si trova nella natura referenziale e riflessiva del discorso a parte. L'autore allarga lo spazio dell'intersoggettività e delle manipolazioni e il lettore si trova in posizione di vantaggio rispetto agli altri personaggi. Alla fine dell'atto l'azione si sposta nell'osteria, davanti alla quale arriva Adalberto, un giovane italiano, che è venuto per fare "affari che si trattano con fuoco” (ibi, 27), ed esalta gli Slavi: “[...] In questi luoghi il buon cuore è proprietà esclusiva degli Slavi a quanto io so" (ibid). Il terzo atto chiude con una scena nella quale il Negro arriva di notte davanti alla casa di fronte all'osteria. Invita altre quattro persone vestite con mantelli neri e con la testa coperta. Uno di loro sale, cerca di aprire la finestra e tira fuori il coltello.

Nel quarto atto veniamo a sapere dell'omicidio di Caterina e del rapimento di Daniza. Paolo, Giorgio, Adalberto, fra Guido, l'oste e il servo speculano sui possibili motivi dell'omicidio di Caterina, sulla gelosia turca, sui beni di Caterina e sulla lussuriosa passione per Daniza. Da un lato pensano alla vendetta, dall'altro si definiscono "amici dell'umanità" (Vidovich, I875, 3I). Fra Guido calma la situazione. La sua fun- zione drammaturgica, infatti, è quella di alleviare le passioni umane primarie, come il desiderio di vendetta, di alleviare sentimenti incompatibili con la fede, generalmente di rallentare l'azione. Questa funzione ha un ruolo anche nelle scene successive di peripezia e risoluzione. Cerca di riconoscere l'universale e il cosmico nella vita di tutti i giorni. Incuriosisce gli altri personaggi quando dice alla fine della scena che potrebbe conoscere la causa della scomparsa di Daniza. Adalberto chiude l'atto: "Sì: ed un evviva agli amici dell'umanità, a qualunque ceto, religione; e razza appartengano!" (ibi, 34 ).

L'azione si sposta nella casa di Mohamed Pascià dove in un dialogo con Ahmed, Mohamed non esprime preoccupazione per l'insurrezione: “[...] Pensa però ch' io avrò importanti affari, e che le cannonate potrebbero darmi fastidio. (ride) Ah! Ah! Un' insurrezione in $\mathrm{Er}-$ zegovina! Farebbe proprio ridere!" (ibi, 35). Fatima racconta a Mohamed e ad Ahmed il caso di Caterina e Daniza. Djemal chiede spiegazioni a Mohamed riguardo alla situazione delle due donne. Egli è offeso dal modo di fare di Djemal e gli dice che comprende lo zelo giovanile, ma che se avesse pronunciato un'altra parola sarebbe stato dimesso dalla sua carica ed imprigionato. La tensione teatrale si accende nel contrasto diretto fra i due uomini. Per Djemal è meglio morire che vivere come un codardo, obbediente a tiranni irragionevoli, e ritiene che il popolo turco abbia diritto all'amore e alla tolleranza. Nel frattempo, si disarma e gli offre la sua spada. Mohamed lo chiama “[...] Novello Pietro d'Amiens, Eremita potresti far sorgere in Europa l'idea di una settima Crociata contro i turchi" (Vidovich, I875, 39). Questa citazione è leggibile in chiave intertestuale in quanto viene nominato Pietro d'Amiens e può essere considerata una citazione implicita, in quanto veicolo di un bagaglio storico che si suppone venga riconosciuto subito dal lettore (Franzini, 2002, 66). Fatima prega per la vita di Djemal, il quale alla fine dell'atto confessa a fra Guido il suo amore verso Daniza. Essendo vicini alla risoluzione si restringe il campo d'azione. Il quinto atto inizia a casa del Pascià. Daniza 
dorme su un divano, vestita in abito turco, senza velo e con i capelli sciolti. È interessante notare che, nell'iconografia cristiana, Maria Maddalena è sempre raffigurata con i capelli lunghi e non legati, come segno di resa a Dio. "(Parla dormendo) Si, si, mia buona madre. Avrò coraggio, avrò pazienza, confiderò nel buon Dio, come tu mi raccomandi. (Svegliandosi lentamente) Ah! Sono sola...! Non c'è mia madre! Pur troppo ho sognato" (ibi, 45). Il suo sogno è in correlazione con gli eventi al primo livello, tramite il quale Vidovich dà spazio alle espressioni liriche. Lei si fida di Dio e l'esperienza del sogno la aiuta in una situazione di crisi. Nonostante il suo elogio alla bellezza e al sentimento delle donne slave, Daniza definisce Mohamed demone e mostro infernale. Lei preferisce versare il suo sangue, piuttosto che dargli la sua anima, ed è determinata nella sua scelta tra essere schiava o regina.

Nel frattempo dal dialogo tra Adalberto e l'oste si evince un'idea di superiorità intellettuale degli italiani sugli Slavi: "Adal. Oh! Beata semplicità dei monti!” (Vidovich I875, 49) che scatena un interessante messaggio di fra Guido ad Adalberto:

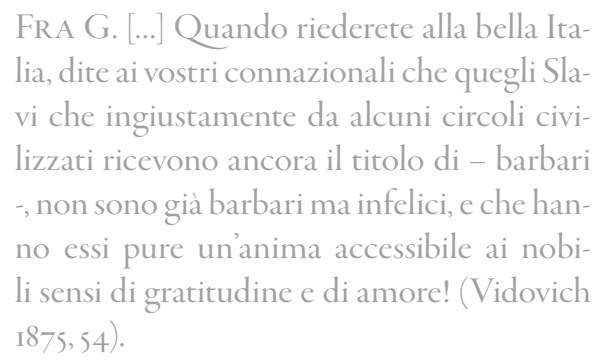

La conversazione, carica di messaggi morali, non assomiglia a una conversazione naturale ed è interrotta da un oste che annuncia l'arrivo di sei capitani, slavi e italiani. Si avverte l'inizio della rivolta. Fra Guido, nella sesta scena in cui è solo con Adalberto, decide che il suo ruolo nella rivolta è di essere padre, fratello e amico di tutti:

Fra G. [...] Nei momenti fatali delle lotte, io non conoscerò, non amerò nessuno a preferenza, ma sarò a tutti ugualmente padre, fratello, amico! L'uomo che si è dedicato al servizio di Dio, deve credere all'immorta- lità delle anime, che sono tutte egualmente emanazioni Divine, dunque, in certi momenti supremi non deve essere né giudice, né parte. Egli deve assistere e pregare, lasciando che Dio solo giudichi! (ibi, 57).

L'atto si chiude con l'incontro tra Fra Guido, Paolo, Giorgio e Djemal che racconta loro l'intera verità su Mohamed e Ahmed.

Il sesto atto viene aperto da Fatima in un monologo lirico, e i suoi pensieri si sintetizzano nella seguente citazione, nella quale offre la chiave di interpretazione del rapimento di Daniza:
(1)

la fiamma voluttuosa nel Mussulmano! Meglio fossero nate senz' occhi! Ci chiamano regine..., e siamo tali nelle ore del piacere, per essere poi per sempre schiave degli schiavi nelle ore della vita! [...] Oh! ma non è possibile che Allah nel crearci, abbia voluto così! Ogni donna sente nel suo cuore lo scopo sublime della propria missione nel mondo che non è certo missione d' inerzia abbietta e servile! (Vidovich I875, 60).

Travestito nell'eunuco Omar, che ogni mattina porta i fiori a Fatima, Djemal va a trovare Fatima e chiede il suo aiuto nella ricerca di Daniza. Lei accetta di darglielo e si schiera a favore dei protagonisti, Djemal e fra Guido, che promuovono l'uguaglianza dei popoli e i diritti umani:
Fatim. [...] Meglio morire pel bene, che vi- vere inerte in mezzo a tesori; meglio morire soccorrendo gli oppressi, che vivere in mez- zo a favolose delizie di un lusso che fa ricor- dare ogni tratto, le giustizie maledizioni di coloro che gemono nel dolore e nella mise- ria, e l'eterno disprezzo degli uomini onesti e forti, che si procurano il sublime e doveroso piacere di soccorrere gl'infelici (ibi, 64).

Djemal riesce a salvare Daniza e a portarla a Paolo. I due si sposano e aiutano Djemal e Fatima a fuggire in Asia. Viene annunciata l'insurrezione e il dramma si chiude con l'incontro tra la Vila e il Destino delle Nazioni che annuncia la pace e ordina alla Vila di osservare la Fede, una 
giovane donna, vestita di bianco e con i capelli lunghi: "FEDE. Io sono la Fede. Questa croce non è simbolo di martirio, ma segnale di tolleranza! Questo fuoco, è fuoco di amore universale. Un giorno tu canterai, o Vila, percorrendo tutte le Slave terre, un inno di gloria pel Supremo Spirito, e quell'inno verrà ripetuto da tutte le nazioni, quando saranno sorelle!" (ibi, 76) Rinnovellando la scena del prologo e i suoi protagonisti, il dramma assume un aspetto astorico e atemporale (Pfister 1998, 387).

\section{II}

Un esempio del testo, Bonaventura Vidovich lo inviò a Giuseppe Garibaldi. Nella risposta datata Is novembre 1875 , inviata da Roma, Garibaldi scrive: "Grazie per il vostro lavoro patriottico L'insurrezione Erzegovese, che leggerò con tanto interesse. Vi stringo la mano e sono Vostro G. Garibaldi." (Berlam 1934) Supponiamo che la ragione per la quale Vidovich avesse inviato il testo a Garibaldi, fosse il manifesto di Garibaldi risalente al 29 agosto 1875 nel quale a Roma dava supporto a Liubibratić e ai suoi compagni (Elia 1904, 427-28). È Paolo che menziona Ljubibratić elencando gli eroi slavi: "Paolo: (Spiega in fretta il foglio, e legge) Che vedo? Michele Bolardich-Ljubibratich! Nome noto, e caro agli Slavi! Luca Petkovich, Tripko Vukalovich, Toma Tomasevich, Rade Bajkovich! Tutti benemeriti Slavi! Ma chi è poi questo Miroslav Hubmayer?" (Vidovich 1875, I4) Inoltre, Garibaldi, dopo aver saputo della sconfitta dei Turchi nella battaglia di Piva, il 5 novembre 1875 , ha inviato da Roma una lettera a Popović, presidente del comitato dei ribelli erzegovesi nella quale scriveva: "Caro Popovich, I liberi d'ogni paese europeo esultano per la splendida vittoria degli eroici figli dell'Erzegovina orientale" (Elia 1904, 429).

L'autore annovera i motivi già affermati nella letteratura drammatica, come il triangolo amoroso tra un tiranno, una vergine e un eroe di guerra, e la scelta dell'onore in guerra invece che dell'amore. Vidovich prosegue con le idee di suo padre, Marco Antonio Vidovich, che bisogna annullare i tiranni e i despoti: "Paolo. [...]
Morte ai tiranni, ai despoti assassini!" (Vidovich I875, 31). Cerca di dare validità storica al dramma rappresentando fedelmente gli Slavi e i Turchi. Indica il loro modo di pensare, di vestire e agire in diverse situazioni: "N.B. ([...] Il saluto alla Turca, che ha per significato: Che la terra mi copra dinanzi a Voi!" (ibi, I4). D'un lato gli Slavi sono rappresentatati come religiosi, e dall'altro come superstiziosi. Ai personaggi turchi assegna nomi tipici, per esempio, Fatima era una delle tre figlie del profeta (Glassé 2006, 153). Tra i personaggi slavi solo Daniza ha un nome slavo, noto nella poesia popolare e nella mitologia slava precristiana, mentre gli altri personaggi slavi hanno nomi italiani (Paolo, Giorgio e Fra Guido). Vidovich persiste in un' idea precisa dell'Italia e degli italiani come coloro che portano la civiltà e il progresso e il cui rappresentante è Adalberto, un giovane italiano?.

Il nucleo portante della trama sono i rapporti contrastanti e corrispondenti indicati già nell'elenco dei personaggi. Nonostante la prima impressione ci faccia pensare di essere di fronte ad un gruppo di personaggi privi di centro, sono poi in realtà Djemal e Mohamed a caricarsi di valenze più drammatiche. D'un lato Fatima e Djemal si rivelano personaggi dinamici, il cui lato affascinante è il loro comportamento antitetico all'immagine stereotipata dei Turchi. Dall'altro lato, Mohamed Pascià manifesta l'alterigia, la vanità e l'abuso di potere messi in luce dagli autocommenti e da uno scavo psicologico accanito, che mette a nudo l'anima del protagonista.

Non a caso il dramma è imperniato sul conflitto slavo-turco. I drammi sociali hanno la caratteristica di attivare opposizioni all'interno di gruppi e classi sociali, trasformando queste posizioni in conflitti, che per essere risolti necessitano una rivisitazione critica di particolari aspetti dell'assetto socioculturale (Turner 1986, I31). Le carenze drammaturgiche, come il ritmo rallentato, i dialoghi abbondanti in messaggi mora-

\footnotetext{
Uno degli esempi è la produzione letteraria di Luigi Fichert (Zara I826 - Venezia I899) nella quale l'autore poliedrico presenta l'Italia come un centro culturale nel quale la missione degli italiani era quella di aiutare il resto del mondo, innanzi tutto gli slavi (Bukvić $2013,4 \mathrm{I}-72$ )
} 
li, le situazioni drammatiche impersuasive, i personaggi piatti, possono essere compensate dallo scopo del testo, più di carattere politico-nazionale che letterario. Vidovich seguiva la moda del tempo e voleva inquadrare nel dramma il piano metafisico-riflessivo e far girare l'intreccio romantico-avventuroso intorno a Daniza, amata da Djemal, il Turco dai sentimenti puri, un vero filantropo, come lo era d'altronde lo stesso Bonaventura Vidovich (Zorić 1961, I87-89).

Costruendo un'immagine positiva del Turco mediante i personaggi di Djemal e Fatima si genera una svolta rispetto alla consolidata struttura socioculturale. La riflessione teorica di Victor Turner è quella che meglio si adatta al riguardo proprio perché i drammi sociali rivelano strati sottocutanei della struttura sociale e fanno affiorare elementi positivi della società stessa (Turner 1986, I3I). Vidovich si è ispirato all'opera di sua madre, Anna Vidovich, che drammatizzò l'amore tra la musulmana e il cristiano nel periodo quando il Turco era il nemico più crudele in assoluto nella letteratura croata risorgimentale. Conviene ricordare il suo dramma lirico Alve ed Ippia, vittime d'amore pubblicato nella raccolta Mestizie e distrazioni a Zara nel 1846 e il poema Bezmišljenja u ljubavi/Le sconsigliatezze in amore del I85I. Niccolò Tommaseo accenna alla Bosnia ed Erzegovina nella terza prosa delle Prose "D’un vecchio calogero": "Chi pensa, o Bosnia, o Erzegovina infelici, chi pensa ai vostri dolori? Come schiavo che in carcere profonda, appena vede il raggio del di simile a crepuscolo notturno, e le grida di lei per le volte tenebrose si perdono, e non giungono all'orecchio dell'uomo; così né voi della colta Europa vedete la luce, e la colta Europa le vostre miserie non sente." (Zorić 1976, 595) Niccolò Battaglini, autore di origine zaratina (Zara 1826 - Venezia 1887) e contemporaneo di Bonaventura Vidovich pubblicò L'Erzegovina, cenno storico a Venezia nel 1875 (Sabalich I887). È da supporre che Luigi Fichert (Zara I826 - Venezia I899), scrittore dalmata e contemporaneo di Vidovich, si ispirasse al dramma di Bonaventura Vidovich nella stesura della tragedia Bielka di Bosnia nel 1876 e del poema La Madre triestina del I879, ma rinunciasse alla veste allegorico-sociale. La Bosnia è stata ampiamente rappresentata anche nella letteratura risorgimentale croata. Il mito della Bosnia come spazio di sofferenza cristiana sotto il dominio turco e il suo declino a causa della divisione etnica slava, da un lato, era una buona base per lo sviluppo delle ideologie slave e anti-turche, e dall'altro, essendo uno spazio esotico, poteva incoraggiare la formazione di diversi componimenti in armonia con la poetica del Romanticismo (Dukić 1998, 318).

\section{Povzetek}

Prispevek prinaša analizo drame Linsurrezione erzegovese. La Slavia - Il mondo Bonaventure Vidovicha, ki je izšla v Livornu leta I875, ko je vladalo posebno zanimanje za bosansko vprašanje; $v$ ta sklop zanimanja se uvršča tudi tragedija Luigija Ficherta Bielka di Bosnia (Bjelka iz Bosne). Vidovich je bil dalmatinskega izvora, sin znanih Anne in Marca Antonia Vidovicha, ki sta zapustila bogato literarno delo v italijanskem jeziku v Dalmaciji v 19. stoletju. Prispevek analizira Vidovichevo preple- $\square$ tanje med literaturo, mitologijo in zgodovino, $s$ katerim želi ustvariti kulturno okolje, ki bi izbrisalo vsako delitev med narodi in ljudstvi kot so Italijani, Slovani in Turki v zapletenem zgodovinskem kontekstu.

\section{Summary}

The contribution analyses the social-allegoric drama Linsurrezione erzegovese. La Slavia - Ilmondo of Bonaventura Vidovich, published in Livorno in I875. It is a period of the particular interest for the Bosnian social and political context, which can be seen also in Fichert's tragedy Bielka di Bosnia. Bonaventura Vidovich is a Dalmatian writer, best known for his parents Anna and Marco Antonio Vidovich, both writers who left a vast literary heritage in Italian and Croatian languages in Dalmatia in the nineteenth century. The contribution tries to highlight how Vidovich uses literature, mythology and history to build a cultural imaginary that erases all limits between nations and peoples such as the Italians, the Slavs and the Turks in a complex historical context.

\section{Riferimenti bibliografici}

Berlam, A. 1934. "Una famiglia di letterati dalmatici del primo ottocento. I 
Conti Vidovich da Sebenico e loro, corrispondenza coi più famosi letterati del loro tempo." La Porta Orientale, 3 (6): 376 391.

Bukvić, A. 2013. "Emancipazione slava nell'opera d'impegno di Luigi Fichert." In Letteratura, arte, cultura tra le due sponde dell'Adriatico, a cura di N. Balić-Nižić, L.

Borsetto, A. Jusup Magazin, 4I-72. Zadar: Sveučilište u Zadru.

Detoni Dujmić, D. 1998. Ljepša polovica književnosti, Zagreb: Matica hrvatska.

Elia, A. 1904. Ricordi di un garibaldino dal I847-48 al I9oo. Roma: Tipografia del Genio Civile.

Franzini, F. 2002. "Citazione invisibile". Leitmotiv 2: 65-69.

Glassé, C. 2006 . Enciklopedija Islama, Sarajevo: Libris d.o.o.

Kaznačić, I. A. 1876. "Dva nova izdanja u talijanskome jeziku u slavjanskome duhu." Vienac 5: 79-80.

Nižić, Ž. 2009. "La storia come gioco poetico nel poema Romolo ossia la fondazione di Roma, di Anna Vidovich." In Tempo e memoria nella lingua e letteratura italiana. Ascoli Piceno, 557-57I.

Pavličević, D. 2000. Kratka politička i kulturna povijest Bosne i Hercegovine. Zagreb: Impress.

Pfister, M. 1998. Drama: teorija i analiza. Zagreb: Hrvatski centar ITI.

Rados, Z. 2007. Hrvatska književnost u Zadru (Ig. st.). Zadar: Thema.

Sabalich, G. 1908-1922. La Cronistoria Aneddotica del Teatro Nobile di Zara (I78II88I). Zadar: A. Nani.

Sabalich, G. 1887. "Niccolò Battaglini". Scintille.

Solar, M. 201. Književni leksikon, Zagreb: Matica Hrvatska.

Tomasović, M. 2009. “Ana Vidovich, Književni profil.” Nova Croatica 3: I-3I.

Turner, V. 1986. Dal rito al teatro. Bologna: Il Mulino.
Vidovich, B. I875. L'insurrezione erzegovese. La Slavia - Il mondo, Livorno: Tip. E Cart. V. D. Ottone.

Vidovich, B. 1878. In morte di S. M. il re d'Italia Vittorio Emanuele II e per l'avvenimento al trono di $S$. M. il re Umberto I, carme. Livorno: Tip. F. Marchetti.

Zorić, M. 1961. Odjeci sa slavenskog Balkana u književnosti $\gg$ Tré́e « Italije. Sarajevo: Naučno društvo NR Bosne i Hercegovine.

Zorić, M. 1970. "Nikola Tommaseo i pjesnikinja Ana Vidovicheva iz Šibenika.” Filologija 6: 335-352.

Zorić, M. I971. Romantički pisci u Dalmaciji na talijanskom jeziku. Zagreb: Rad JAZU.

Zorić, M. 1976. “Le prose „D’un vecchio calogero" di Niccolo Tommaseo." Studia Romanica et Anglica 3: 4I-42.

Zorić, M. 2002. "Ana Vidovich, Marko Antun Vidovich i Nikola Tommaseo u svjetlu neobjavljene prepiske." Hrvatsko-talijanski književni odnosi 3: 367-54I. 